\title{
On-the-fly adaptation of selectivity in the flanker task
}

\author{
Carola Lehle and Ronald Hübner \\ Universität Konstanz, Konstanz, Germany
}

\begin{abstract}
The processing selectivity in the flanker task has been shown to depend on the ratio of congruent trials to incongruent trials in a task (Gratton, Coles, \& Donchin, 1992). If congruent trials are more frequent than incongruent ones, the flankers are more attended and, consequently, the flanker congruency effect is increased. Recent results suggest that participants can even allocate attention on the fly to the flankers - that is, in a highly flexible way after stimulus onset - depending on the frequency of incongruent trials on a certain stimulus location. Because location plays a unique role in stimulus selection, we investigated in two experiments whether selectivity can also be adjusted on the fly depending on stimulus color. The results demonstrate that color can be used for such an adjustment, but only if the association between color and frequency has been previously learned under blocked conditions.
\end{abstract}

Over the past decades, many studies have used conflict paradigms such as the Eriksen flanker task (B. A. Eriksen \& C. W. Eriksen, 1974) or the Stroop task (Stroop, 1935) to investigate the limits of visual selective attention. In these tasks, a target stimulus has to be processed selectively, whereas distractor stimuli have to be ignored. The often observed interference effects indicate that selectivity is not perfect and varies with stimulus factors, such as the discriminability between target and distractors (e.g., Miller, 1991; Paquet \& Craig, 1997). Moreover, there is increasing interest in showing that not only stimulus factors, but also processing strategies, affect selectivity (e.g., Corballis \& Gratton, 2003; Gratton, Coles, \& Donchin, 1992; Hübner \& Lehle, 2007; Jacoby, Lindsay, \& Hessels, 2003; Stürmer, Leuthold, Soetens, Schröter, \& Sommer, 2002; Tzelgov, Henik, \& Berger, 1992; Wendt, Kluwe, \& Vietze, 2008). However, the knowledge about how and when strategies affect selectivity is still scarce. In the present study, we used the Eriksen flanker task to further investigate this question.

In the flanker task, a target stimulus is selected for categorization, while simultaneously present flanker stimuli must be ignored. The flankers can be either congruent or incongruent with the target. Congruent flankers are linked to the same response category as that of the target; incongruent flankers are linked to the response category opposite to the target's. If the flankers are coprocessed, typically the performance with incongruent flankers is impaired relative to performance with congruent ones. This difference in performance, which has been called the flanker congruency effect (FCE), can be used as a measure for selectivity (B. A. Eriksen \& C. W. Eriksen, 1974; C. W. Eriksen \& Schultz, 1979).

The studies showing a strategic influence on the FCE (Corballis \& Gratton, 2003; Gratton et al., 1992; Wendt et al., 2008) suggest that humans apply selection strategies according to the utility principle (i.e., they optimize processing by allocating a specific amount of attention to the flankers depending on their utility). Attending to the flankers is useful for performance if they activate the same response, but detrimental if they activate a different response.

Therefore, the utility principle can be tested by varying the ratio of congruent trials to incongruent trials. If incongruent trials are frequent, flankers should be less attended and, consequently, the FCE should be reduced. In contrast, if congruent flankers are frequent, the flankers should be attended strongly and the FCE should be increased. Indeed, such effects have been observed (Gratton et al., 1992). In addition to these global strategies, which are applied during a whole block of trials, current theories of cognitive control (Botvinick, Braver, Barch, Carter, \& Cohen, 2001; Gratton et al., 1992; Ullsperger, Bylsma, \& Botvinick, 2005) assume that the processing can also be strategically adapted on a trial-by-trial basis, either in response to the conflict on the preceding trial or in response to a cue indicating the upcoming trial type.

However, selection strategies seem to be even more flexible. Recent studies (Crump, Gong, \& Milliken, 2006; Wendt et al., 2008) indicate that participants can adjust the selectivity on the fly after stimulus onset, depending on stimulus location. Wendt et al., for instance, presented stimuli randomly at different locations, where each location was associated with a certain ratio of congruent flankers to incongruent flankers. As a result, they found that the FCE was smaller at the locations where incongruent flankers occurred more frequently. Thus, different degrees of processing selectivity for any two stimulus locations associated with different ratios of congruent stimuli to

C. Lehle, carola.lehle@uni-konstanz.de 
incongruent stimuli seem possible, independent of the visual hemifield (cf. Corballis \& Gratton, 2003).

In general, the location of a stimulus receives priority in processing (e.g., Magen \& Cohen, 2005; Tsal \& Lavie, 1993). Therefore, provided that location can be used for an on-the-fly adaptation of selectivity, the situation can be different for other dimensions, such as color or form. For the standard Stroop task, where selectivity is based on color, Jacoby et al. (2003) demonstrated that stimulus color could be used for an adaptation according to the ratio of congruent trials to incongruent trials. However, in a variant of the Stroop task where the relevant and the irrelevant dimensions were spatially and temporally segregated (Crump et al., 2006), participants were able to use the location, but not the form, of the stimuli to adjust their processing. Because the standard flanker task is a spatial selection paradigm, adaptation might also be possible only in response to stimulus location.

Whether location is indeed unique for an on-the-fly adaptation of selectivity was investigated in the present study, where color was used as an informative dimension in the flanker task. The stimuli could have one of two colors, which were associated with an unequal ratio of congruent flankers to incongruent flankers. However, the overall ratio of congruent trials to incongruent trials within each block was equal. Thus, if spatial attention can generally be allocated on the fly, depending on the expected utility of the flankers, participants should also be able to use the color information accordingly.

\section{EXPERIMENT 1}

In the present study, we used numerals as stimuli and the participants had to judge the parity (even or odd) of the target stimulus in a flanker task. Of the red (or green) stimuli, $80 \%$ were congruent and $20 \%$ incongruent. For the green (or red) stimuli, this proportion was reversed. The overall ratio of congruent trials to incongruent trials within each block was $50 \%$. If stimulus color can be used to adjust the selectivity, flanker processing should be decreased for stimuli whose color indicates a high probability of incongruent flankers, as opposed to stimuli of the other color. This difference should be reflected by the FCE.

\section{Method \\ Participants \\ Twelve students (4 male, 8 female) participated in this experiment either for partial fulfillment of course requirements or for getting paid $€ 5$ per hour. Their age ranged from 21 to 27 years $(M=23.3$ years). All were right-handed (by self-report) and had normal or corrected-to-normal vision.}

\section{Apparatus}

The stimuli were presented on a 21 -in. color monitor with a resolution of $1,280 \times 768$ pixels and a refresh rate of $85 \mathrm{~Hz}$. A personal computer served for controlling stimulus presentation and response registration.

\section{Stimuli}

The stimulus set consisted of the numerals $1-4$ and $6-9$. The height of the stimuli subtended a visual angle of $2^{\circ}$ at a viewing distance of $110 \mathrm{~cm}$, and their width was about $1.36^{\circ}$, depending on the specific digit. The target was always presented centrally with respect to the flankers. The flankers consisted of two copies of a numeral symbol, which were presented left and right of the target at an eccentricity of $1.57^{\circ}$. The target and flankers were always different.

\section{Procedure}

The task for the participants was to judge the parity (odd, even) of the stimuli. They had to press a left key for "even" and a right key for "odd." Each trial started with the appearance of a fixation cross for $400 \mathrm{msec}$. After a blank screen of 600 -msec duration, both target and flankers were presented for $165 \mathrm{msec}$ in red or green on a black background. To increase the overall FCEs, the stimuli were presented with spatial uncertainty (i.e., they were randomly shifted either to the left or to the right side on the screen, so that the interior flanker always appeared centrally).

Altogether, flankers were congruent on half of the trials - that is, they had the same parity as that of the target - and were incongruent on the other half - that is, they had the opposite parity as that of the target. However, the frequencies of congruent and incongruent trials were different depending on the color of the stimuli: For half of the participants, $80 \%$ of the congruent and $20 \%$ of the incongruent stimuli were presented in green, and $80 \%$ of the incongruent and $20 \%$ of the congruent stimuli appeared in red color. This relation was reversed for the other half of the participants.

Thus, the relevant factors were the congruency (congruent or incongruent) of the stimuli and the congruency associated with the stimulus color ( $80 \%$ congruent or $80 \%$ incongruent). Participants had to respond to the target by pushing one of two buttons with the right hand. One second after the last response, the fixation cross for the next trial appeared. Errors were signaled by a tone. After a short practice block, participants performed eight blocks with 80 trials each. The experimental session took about $1 \mathrm{~h}$.

\section{Results}

\section{Response Times}

The latencies of correct responses to the target stimulus were analyzed in an overall two-factor ANOVA for repeated measurements on the factors congruency and color.

The analysis revealed a significant main effect of congruency $[F(1,11)=162, p<.001]$. As can be seen in Figure 1 , the mean response time was $487 \mathrm{msec}$ on congruent and $537 \mathrm{msec}$ on incongruent trials (i.e., there was an overall FCE of $50 \mathrm{msec}$ ). No other effect was significant.

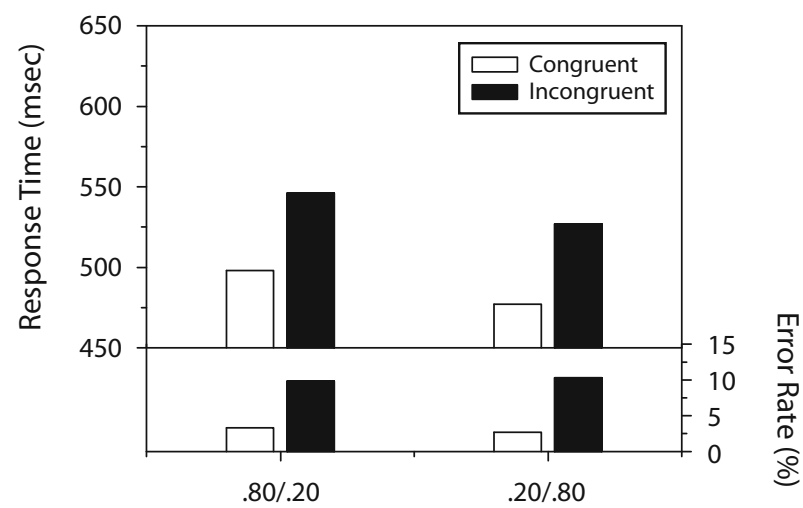

Ratio Congruent/Incongruent (Color)

Figure 1. Mean response times and error rates of Experiment 1 as a function of the congruent/incongruent flanking condition and the ratio of congruent/incongruent trials depending on stimulus color. 


\section{Error Rates}

The error rate in the experiment was $6.53 \%$ overall. The effect of congruency was reliable $[F(1,11)=11.2, p<$ .01] (see Figure 1). The mean error rate on congruent trials was $3.02 \%$; on incongruent trials, it was $10.03 \%$. There was no other significant effect.

\section{Discussion}

In Experiment 1, participants did not adjust their processing selectivity on the fly according to the utility principle. This result contrasts with those of other studies (Gratton et al., 1992; Wendt et al., 2008) where participants allocated attention to the flankers depending on the ratio of congruent trials to incongruent trials. Because it has been demonstrated that selectivity can be adapted in response to stimulus location in the flanker task, one could conclude that this adaptation is not possible in response to stimulus color (see Wendt et al., 2008).

However, a weaker version of this hypothesis also seems conceivable. It could be more difficult to associate flanker utility with a particular color than with a particular location. Thus, participants might be able to allocate attention to the flankers in response to color after increased practice. To assess this possibility, we compared the performance in Block 8 with that in Block 1 and found that there was not even a trend of a practice effect in this respect. However, it is still possible that, because the different color conditions were mixed from the beginning of the experiment, the participants had difficulties in associating the color with a specific ratio of congruent trials to incongruent trials. Whether it is possible to learn the association in blocked conditions was investigated in the next experiment.

\section{EXPERIMENT 2}

In Experiment 2, the participants started with training blocks where the stimulus color and the overall ratio of congruent flankers to incongruent flankers were fixed. As has been shown before (Gratton et al., 1992), if the frequency of congruent and incongruent flankers is unequal and fixed in a block of trials, specific amounts of attention are allocated to the flankers depending on their utility. It could therefore be expected that the participants in Experiment 2 would be able to adjust their selectivity accordingly.

After the training blocks, the same conditions were performed as in Experiment 1. If the participants learned the association between color and processing strategy in the blocked conditions, it seemed possible that they would also apply the color-specific selectivity in blocks where the conditions were mixed.

\section{Method}

Sixteen students (13 female, 3 male) participated in this experiment under conditions similar to those in Experiment 1. Their age ranged from 20 to 34 years ( $M=24.3$ years). All were right-handed (by self-report), all had normal or corrected-to-normal vision, and none had participated in Experiment 1.

Altogether, Experiment 2 replicated Experiment 1, but now specific training was conducted at the beginning. In the training blocks, stimuli of either red or green (balanced across participants) were presented with an unequal and fixed ratio of congruent trials to incongruent trials. Because of the additional blocks, the experiment was conducted in two experimental sessions of about $1 \mathrm{~h}$ each. The two sessions always started with four training blocks, two blocks containing $80 \%$ incongruent and $20 \%$ congruent trials and then two blocks with $80 \%$ congruent and $20 \%$ incongruent trials. Thus, each color was associated with a particular frequency of incongruent trials.

After the training blocks, the mixed blocks were conducted: four blocks per experimental session that were in method, procedure, and experimental factors identical to those of Experiment 1. In the training blocks, the overall frequencies of congruent and incongruent trials were manipulated, so that the factors were congruency (congruent or incongruent) and block (80\% congruent or $80 \%$ incongruent trials).

\section{Results}

\section{Response Times}

Training blocks. First, the latencies of correct responses to the target stimulus were analyzed in an overall two-factor ANOVA for repeated measurements on the congruency and block factors.

The analysis revealed a significant main effect of congruency $[F(1,15)=56.4, p<.001]$. The mean response time was $530 \mathrm{msec}$ on congruent and $593 \mathrm{msec}$ on incongruent trials (i.e., there was an overall FCE of $63 \mathrm{msec}$ ).

The two-way interaction between the congruency and block factors was significant $[F(1,15)=20.2, p<.001]$. As can be seen in Figure 2, the FCE was substantially higher during the blocks with $80 \%$ congruent and $20 \%$ incongruent trials $(107 \mathrm{msec})$ than during the blocks with $80 \%$ incongruent and $20 \%$ congruent trials (19 msec).

Mixed blocks. As in Experiment 1, the latencies of correct responses to the target stimulus were analyzed in an overall two-factor ANOVA for repeated measurements on the factors congruency (congruent or incongruent) and color ( $80 \%$ congruent or $80 \%$ incongruent).

The analysis revealed a significant main effect of congruency $[F(1,15)=30.6, p<.001]$. As can be seen in Figure 3, there was an overall FCE of $54 \mathrm{msec}$. Further-

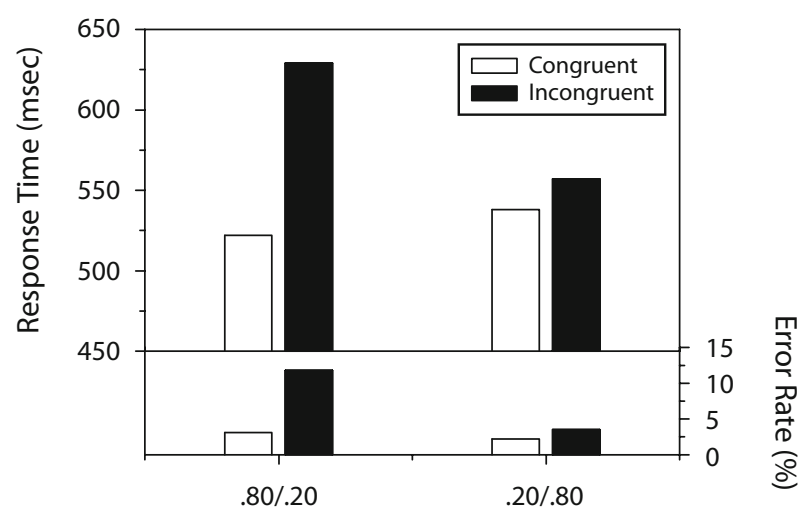

Ratio Congruent/Incongruent (Blocked)

Figure 2. Mean response times and error rates of the training blocks of Experiment 2. The results are shown as a function of the congruent/incongruent flanking condition and the blocked ratio of congruent/incongruent trials. 
more, the two-way interaction between the congruency and color factors was significant $[F(1,15)=6.39, p<$ $.05]$, as can be seen in Figure 3 .

\section{Error Rates}

Training blocks. The error rate in the training blocks of Experiment 2 was on average $4.92 \%$. There was a significant effect of congruency $[F(1,15)=19.4, p<.001]$ (see Figure 2). The mean error rate on congruent trials was $2.19 \%$, and on incongruent trials, it was $7.64 \%$. Furthermore, block produced a reliable effect $[F(1,15)=$ $21.6, p<.001$ ], indicating that more errors occurred in the blocks with frequent congruent trials than in those with frequent incongruent trials $(6.96 \%$ vs. $2.86 \%)$. Finally, there was a significant two-way interaction between block and congruency $[F(1,15)=19.4, p<.001]$. The error rate was particularly high on incongruent trials presented within blocks with frequent congruent trials $(11.8 \%)$.

Mixed blocks. The mean error rate in the mixed blocks of Experiment 2 was $4.43 \%$. Congruency produced a significant main effect $[F(1,15)=28.2, p<.001]$ (see Figure 3). On incongruent trials, errors occurred on $5.93 \%$ of trials, whereas only $2.94 \%$ of trials were incorrect on congruent trials.

\section{Discussion}

In the blocked conditions of the training, participants varied the amount of attention allocated to the flankers depending on their utility and on the frequency of incongruent trials in a block. Furthermore, the training also had the expected effect on the mixed blocks, where color was associated with a particular ratio of congruent flankers to incongruent flankers. Unlike in Experiment 1 , the FCE in response times was now larger for stimuli whose color indicated a high probability of being congruent than for stimuli whose color signaled a high probability of being incongruent. An ANOVA with experiment as the between-participants factor revealed

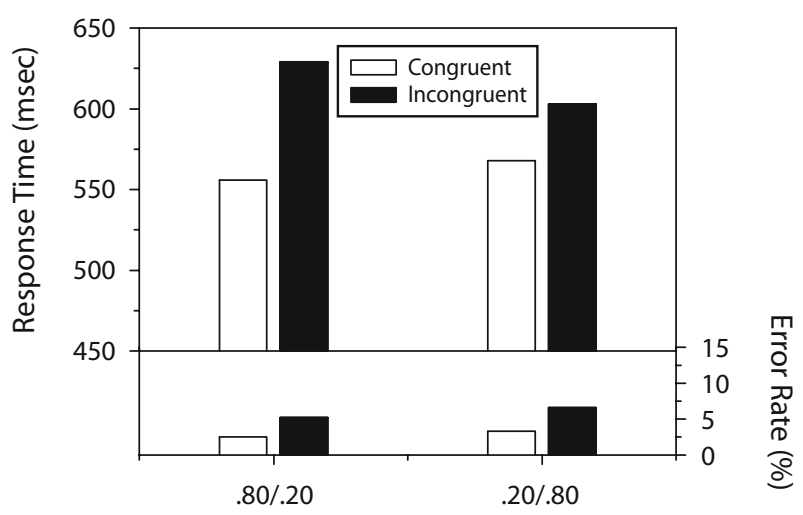

Ratio Congruent/Incongruent (Color)

Figure 3. Mean response times and error rates of the mixed blocks of Experiment 2. The results are depicted as a function of the congruent/incongruent flanking condition and the ratio of congruent/incongruent trials depending on stimulus color. that this difference was significant $[F(1,26)=4.61, p<$ $.05]$. Obviously, the training where stimulus color and processing strategy were fixed enabled participants to use the color for on-the-fly adjustments of the amount of flanker processing.

\section{GENERAL DISCUSSION}

In the present study, we investigated the limits of adjusting processing strategies in the flanker task. In previous studies, it has been shown that participants adapt their processing selectivity according to the utility principle of strategy selection (Crump et al., 2006; Gratton et al., 1992; Jacoby et al., 2003; Ullsperger et al., 2005; Wendt et al., 2008). The utility principle states that participants allocate more attention to the flankers if they expect a congruent trial instead of an incongruent trial.

Until now, several methods have been applied to test the utility principle, each of which requires a more or less dynamic adaptation of processing selectivity. A consistent strategic adjustment of processing selectivity can be observed if the ratio of congruent trials to incongruent trials is unequal in a block of trials (Gratton et al., 1992). Furthermore, there is evidence that selectivity can also be adapted on a trial-by-trial basis (Gratton et al., 1992; Ullsperger et al., 2005).

However, within certain limits, the processing strategies seem to be even more flexible. As we have shown, participants can adjust their selectivity on the fly after stimulus onset, depending on stimulus color. In Experiment 1 , congruent and incongruent trials were presented with equal frequency. The color of the stimuli, though, was informative about whether the flankers were more likely to be congruent or incongruent. However, in Experiment 1 , participants did not adjust their selectivity in response to the color. The FCE was identical for the two conditions.

Therefore, in Experiment 2, we examined the hypothesis that participants have difficulties in associating the stimulus color with a specific ratio of congruent trials to incongruent trials. We hypothesized that the association might be learned more easily if the different frequencies were presented in a blocked mode. Therefore, in Experiment 2, participants started with training blocks where the overall ratio of congruent flankers to incongruent flankers was unequal and fixed. In these blocks, participants adjusted the processing selectivity according to the frequency of incongruent trials.

More importantly, the training had the expected effect. After the training blocks, the participants performed the same conditions as in Experiment 1. As a result, they were now able to use stimulus color for a strategic adjustment. The FCE was significantly larger for stimuli whose color indicated a high probability of being congruent than for stimuli whose color signaled a high probability of being incongruent.

Altogether, our results show that, for the flanker task, participants are able to adjust their spatial selectivity on the fly, not only in response to stimulus location (Wendt et al., 2008), but also in response to stimulus color. How- 
ever, the results also demonstrate that color can be used in this task only if the association between color and processing strategy was learned previously in blocked conditions. This raises the following question: Under which conditions is training necessary to adjust the processing selectivity after stimulus onset?

Without specific training, adjustments in response to stimulus location have been observed in the flanker task and in a variant of the Stroop task where the relevant and the irrelevant dimensions were segregated spatially (Crump et al., 2006; Wendt et al., 2008), whereas adjustments in response to stimulus color occurred, up to now, only in the standard Stroop task (Jacoby et al., 2003). This indicates that the type of task is decisive in this respect and suggests that strategic adjustments without training are possible in response to the dimension that is relevant for the selection in a task (e.g., location in the flanker task or color in the Stroop task). However, for a definitive answer, further research will be required in order to investigate the influence of training in the different types of tasks.

Apart from our assumption that on-the-fly adjustment of selectivity is triggered by a central mechanism (cf. Cohen, Dunbar, \& McClelland, 1990), there is an alternative account that proposes an automatic control on a single-item level (see, e.g., Logan, Zbrodoff, \& Williamson, 1984; Musen \& Squire, 1993). For instance, participants could learn that the green flanker " 7 " is usually congruent to the target and thus associate it with the response "right key," whereas the opposite response would hold for the red flanker "7." Depending on their color, flankers then trigger different responses automatically in the absence of any strategic allocation of attention. Although such an influence can hardly be ruled out (cf. Jacoby et al., 2003; Wendt et al., 2008), it would have been rather improbable in the present study, because we used a relatively large stimulus set. Furthermore, there is no reasonable explanation why associations on a singleitem level could be learned only in blocked conditions. Associations are probably established on a more general level (i.e., between color and selectivity; cf. Crump et al., 2006).

In conclusion, the present results indicate that the human information processing system can adjust its processing selectivity in a highly flexible way, but only within certain limits. Not all stimulus features seem to be equally effective in this respect. Furthermore, learning might be required for an adjustment of processing selectivity, an issue that has rarely been investigated.

\section{AUTHOR NOTE}

We thank Trammell Neill, Joseph Tzelgov, and Mike Wendt for their helpful comments on an earlier version of this article. Correspondence should be addressed to C. Lehle, Universität Konstanz, Fachbereich Psychologie, Fach D29, D-78457 Konstanz, Germany (e-mail: carola .lehle@uni-konstanz.de).

\section{REFERENCES}

Botvinick, M. M., Braver, T. S., Barch, D. M., Carter, C. S., \& Cohen, J. D. (2001). Conflict monitoring and cognitive control. Psychological Review, 108, 624-652.

Cohen, J. D., Dunbar, K., \& McClelland, J. L. (1990). On the control of automatic processes: A parallel distributed processing account of the Stroop effect. Psychological Review, 97, 332-361.

Corballis, P. M., \& Gratton, G. (2003). Independent control of processing strategies for different locations in the visual field. Biological Psychology, 64, 191-209.

Crump, M. J. C., Gong, Z., \& Milliken, B. (2006). The contextspecific proportion congruent Stroop effect: Location as a contextual cue. Psychonomic Bulletin \& Review, 13, 316-321.

EriKsen, B. A., \& ERIKSEN, C. W. (1974). Effects of noise letters upon the identification of a target letter in a nonsearch task. Perception \& Psychophysics, 16, 143-149.

ERIKSEN, C. W., \& SchUlTZ, D. W. (1979). Information processing in visual search: A continuous flow conception and experimental results. Perception \& Psychophysics, 25, 249-263.

Gratton, G., Coles, M. G., \& Donchin, E. (1992). Optimizing the use of information: Strategic control of activation of responses. Journal of Experimental Psychology: General, 121, 480-506.

HÜBNER, R., \& LEHLE, C. (2007). Strategies of flanker coprocessing in single and dual tasks. Journal of Experimental Psychology: Human Perception \& Performance, 33, 103-123.

JaCoBY, L. L., LindSAY, D., \& Hessels, S. (2003). Item-specific control of automatic processes: Stroop process dissociations. Psychonomic Bulletin \& Review, 10, 638-644.

Logan, G. D., Zbrodoff, N. J., \& Williamson, J. (1984). Strategies in the color-word Stroop task. Bulletin of the Psychonomic Society, 22, 135-138.

Magen, H., \& CoHEN, A. (2005). Location specificity in response selection processes for visual stimuli. Psychonomic Bulletin \& Review, 12, 541-548.

MiLler, J. (1991). The flanker compatibility effect as a function of visual angle, attentional focus, visual transients, and perceptual load: A search for boundary conditions. Perception \& Psychophysics, 49, 270-288

Musen, G., \& Squire, L. R. (1993). Implicit learning of color-word associations using a Stroop paradigm. Journal of Experimental Psychology: Learning, Memory, \& Cognition, 19, 789-798.

Paquet, L., \& Craig, G. L. (1997). Evidence for selective target processing with a low perceptual load flankers task. Memory \& Cognition, 25, 182-189.

Stroop, J. R. (1935). Studies of interference in serial verbal reactions. Journal of Experimental Psychology, 18, 643-662.

Stürmer, B., Leuthold, H., Soetens, E., Schröter, H., \& SomMER, W. (2002). Control over location-based response activation in the Simon task: Behavioral and electrophysiological evidence. Journal of Experimental Psychology: Human Perception \& Performance, 28, 1345-1363.

Tsal, Y., \& LaVIE, N. (1993). Location dominance in attending to color and shape. Journal of Experimental Psychology: Human Perception \& Performance, 19, 131-139.

Tzelgov, J., HeniK, A., \& Berger, J. (1992). Controlling Stroop effects by manipulating expectations for color words. Memory \& Cognition, 20, 727-735

Ullsperger, M., Bylsma, L. M., \& Botvinick, M. M. (2005). The conflict adaptation effect: It's not just priming. Cognitive, Affective, \& Behavioral Neuroscience, 5, 467-472.

Wendt, M., Kluwe, R. H., \& Vietze, I. (2008). Location-specific versus hemisphere-specific adaptation of processing selectivity. Psychonomic Bulletin \& Review, 15, 135-140.

(Manuscript received September 30, 2007; revision accepted for publication March 18, 2008.) 